\title{
Tracing local and regional clusters of carbapenemase- producing Klebsiella pneumoniae ST512 with whole genome sequencing, Finland, 2013 to 2018
}

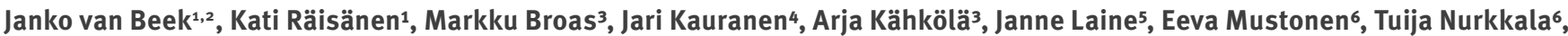
Teija Puhto7, Jaana Sinkkonen ${ }^{5}$, Senja Torvinen', Tarja Vornanen, ${ }^{8}$, Risto Vuento ${ }^{9}$, Jari Jalava ${ }^{1}$, Outi Lyytikäinen ${ }^{1}$

1. Department of Health Security, National Institute for Health and Welfare, Helsinki, Finland

2. European Programme for Public Health Microbiology Training (EUPHEM), European Centre for Disease Prevention and Control (ECDC), Stockholm, Sweden

3. Infection-hospital hygiene unit, Lapland Central Hospital, Rovaniemi, Finland

4. NordLab, Oulu, Finland

5. Department of Infectious Diseases, Tampere University Hospital and University of Tampere, Faculty of Medicine and Life Sciences, Tampere, Finland

6. Hospital hygiene and infectious diseases unit, Länsi-Pohja Central Hospital, Kemi, Finland

7. Department of Infection Control, Oulu University Hospital, Oulu, Finland

8. Kainuu Central Hospital, Kajaani, Finland

9. FimLab laboratories, Tampere, Finland

Correspondence: Outi Lyytikäinen (outi.lyytikainen@thl.fi)

Citation style for this article:

van Beek Janko, Räisänen Kati, Broas Markku, Kauranen Jari, Kähkölä Arja, Laine Janne, Mustonen Eeva, Nurkkala Tuija, Puhto Teija, Sinkkonen Jaana, Torvinen Senja, Vornanen Tarja, Vuento Risto, Jalava Jari, Lyytikäinen Outi. Tracing local and regional clusters of carbapenemase-producing Klebsiella pneumoniae ST512 with whole genome sequencing, Finland, 2013 to 2018. Euro Surveill. 2019;24(38):pii=1800522. https://doi.org/10.2807/1560-7917.ES.2019.24.38.1800522

Background: Two epidemiologically-unrelated clusters of Klebsiella pneumoniae carbapenemase (KPC)producing $K$. pneumoniae were detected among several healthcare facilities (HCF) in Finland by routine surveillance using whole genome sequencing (WGS).

Aim: The objective was to investigate transmission chains to stop further spread of the responsible strain. Methods: In this observational retrospective study, cases were defined as patients with $K$. pneumoniae KPC-3 sequence type (ST)512 strain detected in Finland from August 2013 to May 2018. Environmental specimens were obtained from surfaces, sinks and toilets in affected wards. WGS was performed on $K$. pneumoniae cultures using Illumina MiSeq platform and data were analysed using Ridom SeqShere software $K$. pneumoniae core genome multilocus sequence typing (cgMLST) scheme. Epidemiological information of the cases was provided by HCFs. Results: We identified 20 cases in six HCFs: cluster 1 included 18 cases in five HCFs and cluster 2 two cases in one HCF. In cluster 1 , a link with a foreign country was unclear, 6/18 cases without overlapping stay had occupied the same room in one of the five HCFs within $>3$ years. In cluster 2 , the index case was transferred from abroad, both cases occupied the same room 8 months apart. A strain identical to that of the two cases in cgMLST was isolated from the toilet of the room, suggesting a clonal origin. Conclusions: The clusters were mostly related to case transfer between facilities and likely involved environmental transmission. We show that CPE surveillance using WGS and collaboration between hospitals are crucial to identify clusters and trace transmission chains.

\section{Introduction}

Carbapenemase-producing Enterobacteriaceae (CPE) pose a notable threat to patients and healthcare systems in Europe and globally [1-3]. Infections caused by CPE are associated with high mortality, primarily due to delays in administration of effective treatment and the limited availability of treatment options. CPE are predominantly hospital-acquired and carbapenem resistance is transmitted via clonal spread or horizontal plasmid-mediated transmission [4,5]. Whole genome sequencing (WGS) has recently become the gold standard for bacterial typing and has previously shown to be useful to trace back transmission chains and reveal unexpected transmission routes within outbreaks [6-9].

Inter-regional spread or an endemic situation of CPE was reported by 13 of 38 (34\%) European countries in 2015 [10]. Currently CPE are endemic in Greece, Italy and Turkey, posing a threat of cross-border spread during international patient transfers [3]. CPE are rare in Finland and have been detected sporadically, mostly related to hospitalisation abroad [11]. Only one outbreak has been detected so far when nine patients were found colonised by Klebsiella pneumoniae carbapenemase (KPC)-producing K. pneumoniae (KPC-KP) in a primary care hospital in Southern Finland in 2013 [12]. 
Here we describe how we traced back transmission chains in a single hospital cluster with two K. pneumoniae $\mathrm{KPC}-3$ sequence type (ST)512 patients in Western Finland, and the first regional cluster with $18 \mathrm{~K}$. pneumoniae KPC-3 ST512 patients in five healthcare facilities (HCF) in Northern Finland using WGS. We also report the results of patient screening and environmental investigations related to these clusters.

\section{Methods}

\section{Population and surveillance}

In Finland (population 5.5 million in 2018), publicly funded healthcare is organised in 20 geographically and administratively defined healthcare districts with a population range from 44,000 to 1.6 million inhabitants. Secondary care is provided by 15 central hospitals and five university hospitals additionally provide tertiary care services. Primary care is provided locally by public healthcare centres.

All Finnish clinical microbiological laboratories electronically notify all isolates with reduced susceptibility to carbapenems to the National Infectious Disease Registry and send bacterial strains to the Expert Microbiology Unit of the National Institute for Health and Welfare for confirmation and national surveillance. Since 2015 all CPE strains have routinely been sequenced using WGS and older isolates (2009-14) have been sequenced retrospectively.

\section{National guidelines for control of multidrug- resistant bacteria}

The national guidelines for controlling multidrugresistant (MDR) bacteria cover CPE [13]. According to the guidelines all high-risk patients (defined as patients who either were transferred from a foreign hospital or hospitalised abroad during the previous year) are placed in single rooms, managed by contact precautions like CPE-positive patients, and screened (twice 24 hours apart) for MDR bacteria at the time of admission. When CPE patients are newly identified, potentially exposed contact patients, such as current roommates, are screened and those exposed patients who are already discharged are flagged in the patient's records to institute infection control measures and screening in case of re-admission. More extensive screening for $\mathrm{CPE}$, like point prevalence surveys on wards or units, are considered if positive patients are found during the contact screening. When patients are transferred to other facilities, receiving facilities are informed about the patient's infection or colonisation caused by CPE.

\section{Case definition and epidemiological investigation}

Based on WGS national surveillance analysis, cases were defined as patients with specimens dated from August 2013 onwards with a positive culture of $K$. pneumoniae KPC-3 ST512 belonging to one of two whole genome sequence clusters (GenBank accession numbers cluster1 and 2: SAMN10791377 and SAMN10791378, respectively) with 10 or less allele differences in core genome multilocus sequence typing (cgMLST) analysis. This cut-off was experimentally determined and has been used in similar studies previously [14]. National (public health microbiologists and epidemiologists) and regional (clinical microbiologists, infection control nurses and infectious disease specialists) experts formed the outbreak investigation team. Background information (hospital stay, ward specialty, underlying disease/condition, antimicrobial treatment, clinical outcome, hospital transfers, and travel history) of cases, and patient and environmental screening results were provided by the respective HCF (HCFA: 615 beds; HCFB: 151 beds; HCFC: 113 beds; HCFD: 218 beds; HCFE: 40 beds; HCFF: 779 beds). Data were analysed using descriptive statistics.

\section{Patient and environmental microbiological testing}

Environmental specimens (surfaces, sinks, toilets) were taken with sterile, cotton-tipped swabs. Patient and environmental specimens were plated on selective chromogenic KPC plates (CHROMagar, Paris, France) to screen for MDR Enterobacteriaceae. Isolates were characterised by matrix-assisted laser desorption/ionisation time-of-flight (MALDI-TOF) mass spectrometry (VITEK MS, bioMeriéux, Marcy-L'Etoile, France), and antimicrobial susceptibilities were assessed by disk diffusion method according to clinical breakpoints as published by the European Committee on Antimicrobial Susceptibility Testing [15], or by gradient minimum inhibitory concentrations (MIC) determination test (Etest, bioMeriéux, Marcy-L'Etoile, France). The carbapenemase gene was determined by multiplex real-time PCR for isolates with reduced susceptibility to any carbapenem as per the national guideline $[13,16]$.

\section{Whole genome sequencing}

WGS was implemented for all CPE positive strains. Sequencing was performed on a MiSeq instrument (Illumina, San Diego, California (CA), United States (US)). For the library preparation $1 \mathrm{ng}$ purified DNA was used with a NexteraXT V2 DNA sample preparation kit (Illumina) and paired-end sequenced with a $2 \times 150 \mathrm{bp}$ kit (Illumina). Libraries were scaled to reach 100 -fold coverage. Read mapping analysis was performed from the FASTQ files. Files were transferred to the Centre for Scientific Computing (CSC) environment where sequences were trimmed with Trimmomatic (version 0.33), quality was verified with FastQC (version 0.11.6), resistance genes and multilocus sequence typing (MLST) were analysed with SRST2 version 0.2.0 [17,18]. Commercial software SeqSphere+(Ridom GmbH, Münster, Germany) and the available cgMLST scheme for $K$. pneumoniae from Ridom, were used to prepare the minimum spanning tree.

\section{Ethical statement}

The Finnish Ministry of Social Affairs and Health determined that no ethics committee review was required for 


\section{FIGURE 1}

Minimum spanning tree of core genome sequences of Klebsiella pneumoniae carbapenemase-3 producing K. pneumoniae ST512 isolated from cases, Finland, 2013-2018 ( $\mathrm{n}=20$ cases)

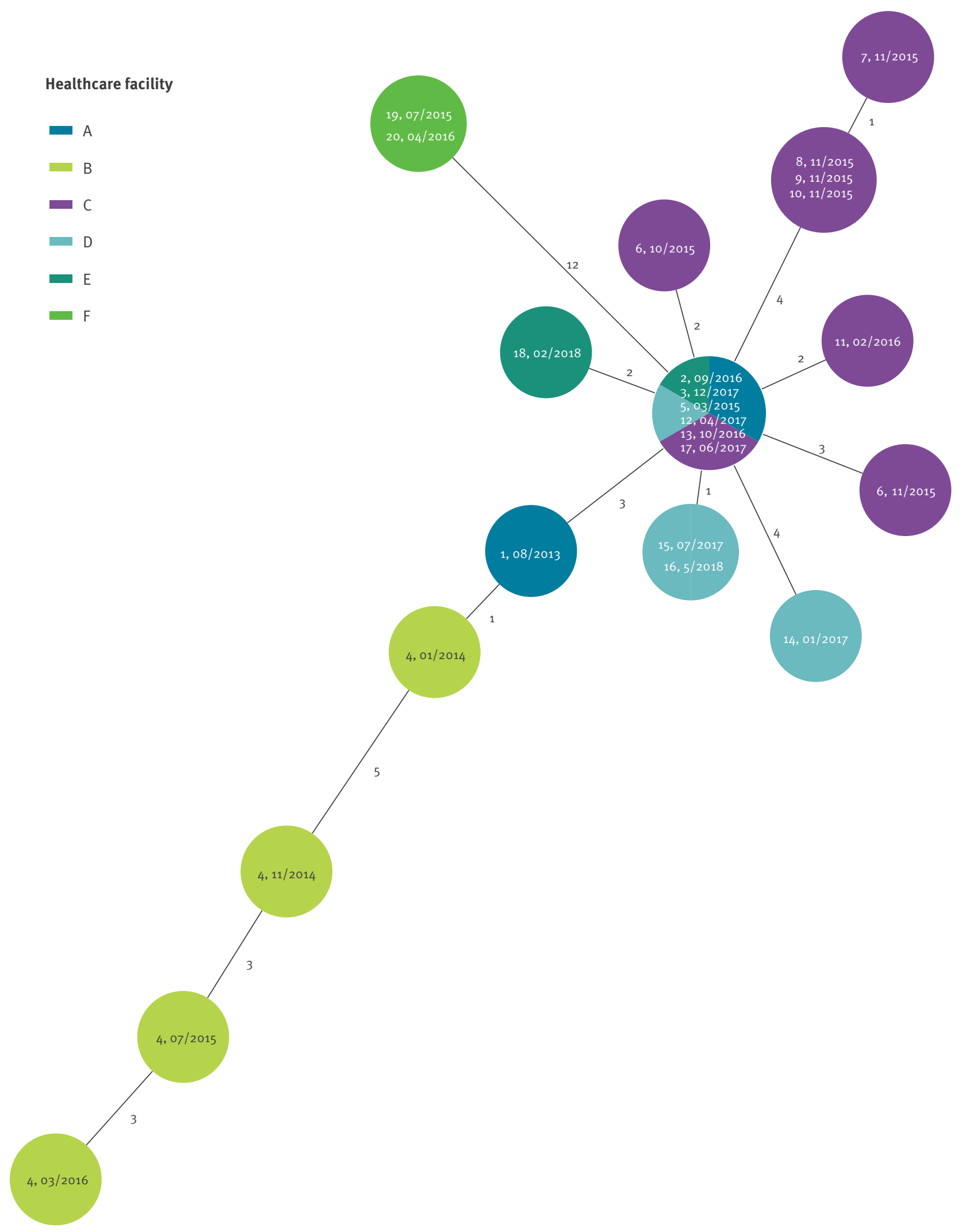

ST: sequence type.

Each circle (node) represents one or multiple identical sequences. The number between the nodes indicates the number of allele differences. The text in each circle indicates the case identifier, sample month/sample year, and the colour indicates the healthcare facility (A-F). 


\section{FIGURE 2}

Distribution over time of Klebsiella pneumoniae

carbapenemase- 3 producing K. pneumoniae ST512 cases,

Finland, 2013-2018 ( $\mathrm{n}=20$ cases)

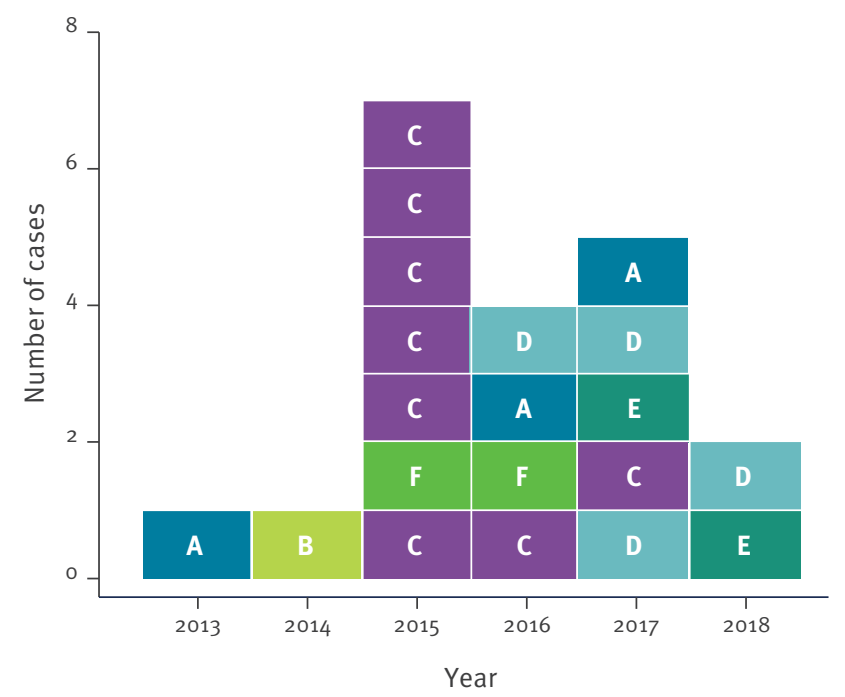

ST: sequence type.

Letters inside boxes indicate the healthcare facility (A-F).

this study since it was conducted as part of an investigation in response to an acute public health problem. Collection of CPE strains is based on the communicable disease law.

\section{Results}

\section{Outbreak detection}

Two separate clusters of closely related (less than 10 allele difference) core genome sequences of $K$. pneumoniae KPC-3 ST512 were identified in a minimum spanning tree analysis of the national surveillance data (Figure 1). The first cluster included 18 cases distributed regionally over five HCFs (HCFA-E) in Northern Finland between August 2013 and May 2018 (Figure 2 and 3). The second cluster included two cases in HCFF between July 2015 and April 2016. The causative strain of the first cluster was only susceptible to ceftazidime/avibactam, colistin and gentamicin and the strain of the second cluster to gentamicin and colistin (not tested to ceftazidime/avibactam).

\section{Description of cases and transmission chains}

The median age of the 20 cases was 69years (range: 30-89years), 15 were male, and all but two had at least one underlying disease. Sixteen cases were found through positive results in clinical specimens (i.e. specimens requested by the attending physician due to clinical signs and symptoms of the patient) and four in routine or enhanced screening. Eight cases received antimicrobials for a clinical infection caused by K. pneumoniae KPC-3 ST512 and one patient died attributable to the infection. A summary of patient information is presented in the Table.

\section{Cluster 1}

The index case (case 1) of the first cluster was a patient with trauma and a drug abuse history. Recent travel history abroad was unclear. This case was treated in HCFA and detected positive by a clinical specimen in August 2013. Case 1 had previously been found negative during the same hospitalisation period when screened for MDR bacteria at intensive care unit (ICU) admission in July 2013 and also weekly according to the routines surveillance cultures of the ICU. Cases 2 and 3 in HCFA were found by positive clinical specimens in September 2016 and December 2017 and had been treated 33 months and 50 months later in the same room as case 1 after the ICU care, respectively.

The strain spread to HCFB via case 4 who had had a preceding hospitalisation in HCFA in the same room, without overlapping stay, as case 1 and was subsequently transferred to HCFB. Case 4 remained positive for the outbreak strain for more than 2 years, but no further transmission was detected.

Case 5 , the first one in HCFC, was identified by a positive clinical specimen in March 2015. No direct epidemiological link between case 5 and any previous cases or HCFA was found. Case 6 in HCFC was identified by a positive clinical specimen 6 months after case 5 and likely obtained the strain via case 5 as they both were treated in wardX even though there was no overlapping stay or common room. Cases 8, 9 and 10 in HCFC were identified by ward screening in November 2015. Case 8 likely obtained the strain via case 5 since both were treated in wardX with overlapping stay, though they did not share a common room. Based on the sequence analysis, cases 9 and/or 10 likely obtained the strain from case 8. Cases 9 and 10 had shared a room in ward $\mathrm{Y}$, which had also been occupied by case 7 but without overlapping stay. Although case 7 was identified by a positive clinical specimen four days before cases 8,9 and 10, sequence analysis revealed that case 7 most likely obtained the strain via case 9 or 10. Case 11 in HCFC was identified by a clinical specimen in February 2016 and had a strain with a sequence most likely derived from case 5 , but no epidemiological link between these cases was found. Case 12 in HCFC was identified by a clinical specimen in April 2017 and had a strain with an identical sequence as case 5 . Both cases (case 5 and 12) had attended weekly haemodialysis in HCFC. Case 12 was previously found negative in the screening conducted after case 5 was found, but thereafter extended-spectrum beta lactamases (ESBL)producing bacteria were detected several times in clinical specimens before the detection of $K$. pneumoniae KPC-3 ST512.

The strain spread to HCFD by cases 13 and 14 who were identified by clinical specimens in October 2016 and January 2017 respectively. Both cases had stayed in 
Epidemiological links between Klebsiella pneumoniae carbapenemase-3 producing K. pneumoniae ST512 cases, Finland, 2013-2018 ( $\mathrm{n}=20$ cases)

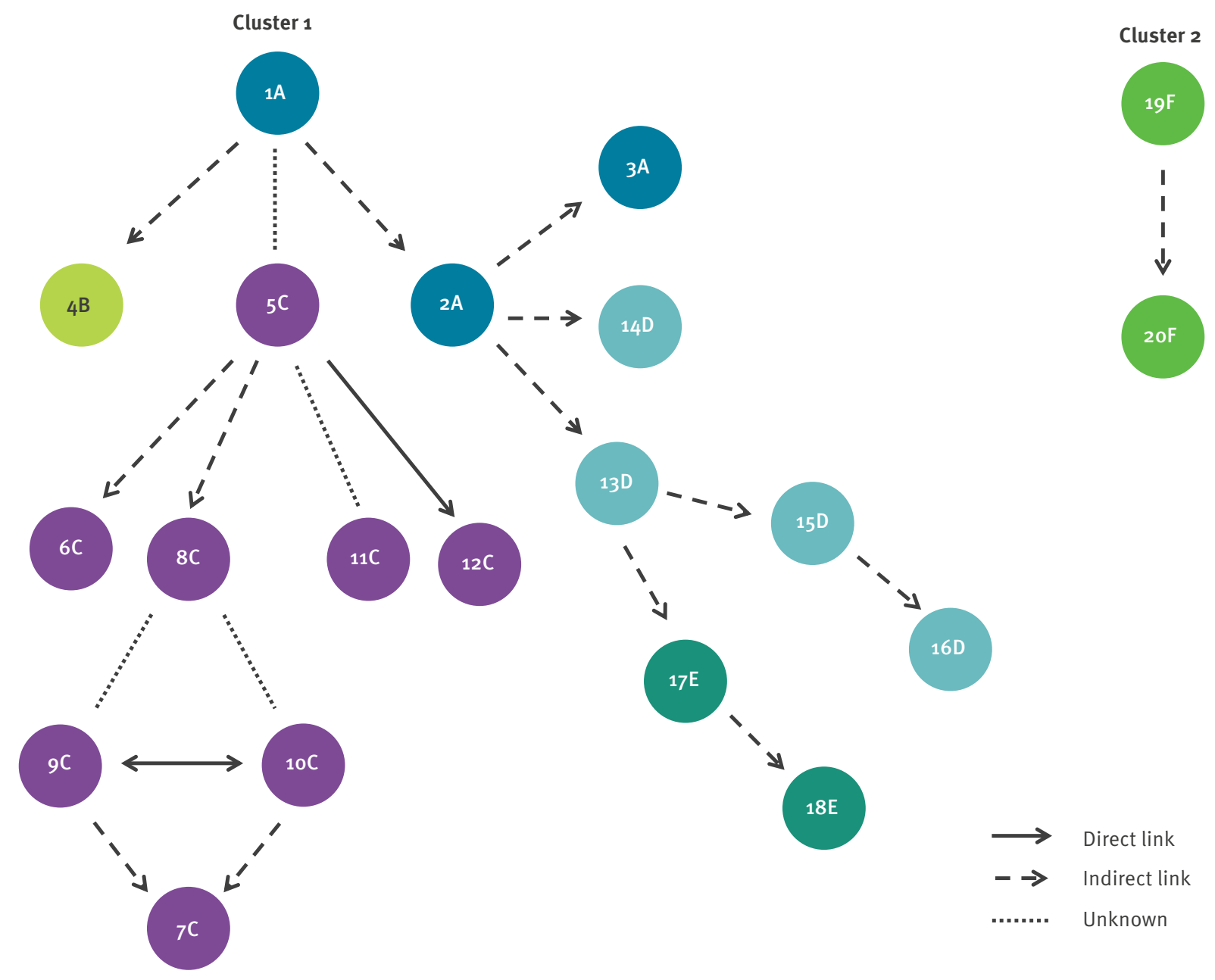

ST: sequence type.

Nodes represent cases (1-20) and letters healthcare facilities (A-F). Arrows indicate epidemiological link between cases, directly (patient-topatient) or indirectly (environmental source or via undetected intermediate patient).

Bidirectional arrows between two cases indicate that one case could have transmitted the strain to another or vice-versa.

the same room as cases 1,2 , and 4 in HCFA (June 2016 to September 2016 and October 2016, respectively) and were subsequently transferred to HCFD. Case 15 in HCFD was identified by a positive clinical specimen in July 2017 and most likely got the strain via case 13 in the same ward who occupied the same room but without overlapping stay. Case 16 in HCFD was identified in May 2018. This case had a strain with an identical sequence as case 15 , and a preceding hospitalisation in HCFD in the same ward and common room as case 15 but without overlapping stay.

The strain was introduced to HCFE in June 2017 by case 17 (found by a positive clinical specimen) who had a strain with an identical sequence and had a preceding hospitalisation in HCFD in the same ward and in a common room as case 13 but without overlapping stay. Case 17 was subsequently transferred to HCFE. Case 18 in HCFE was identified in February 2018 by a positive clinical specimen and obtained the strain in HCFE via case 17 in the same ward who occupied the same room but without overlapping stay.

The median time interval between the first positive specimens of all cases in cluster 1 was 3.0 months (range: 0-15 months).

\section{Cluster 2}

The second cluster consisted of two cases (case 19 and 20) identified in HCFF with an identical sequence 
Characteristics of cases with Klebsiella pneumoniae carbapenemase-3 producing K. pneumoniae ST512 by healthcare facility and first positive specimen, Finland, 2013-2018 ( $\mathrm{n}=20$ cases)

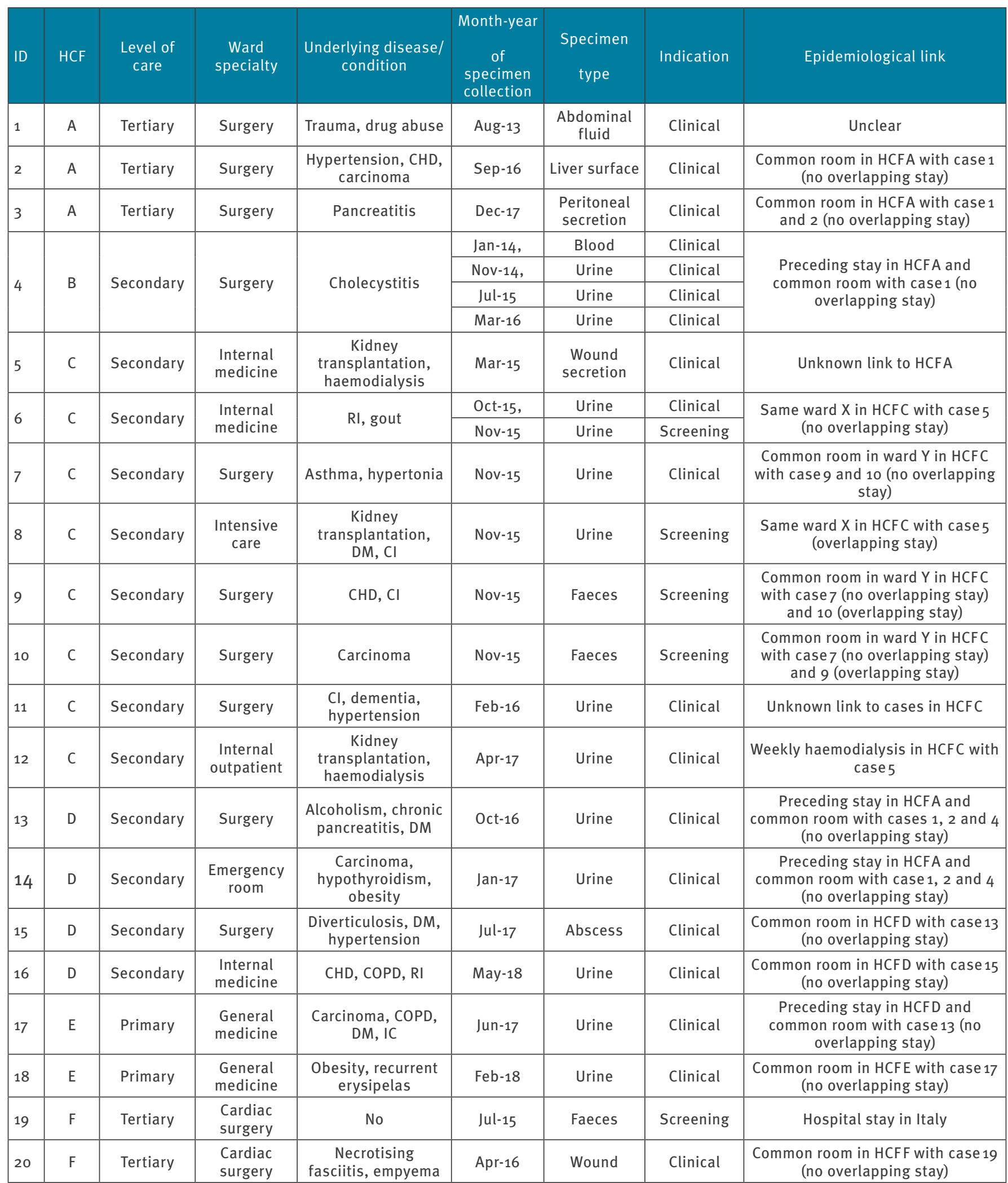

CHD: coronary heart disease; CI: cardiac insufficiency; COPD: chronic obstructive pulmonary disease; DM: diabetes mellitus; HCF: healthcare facility; IC: insufficientia cordis; ID: identifier; RI: renal insufficiency; ST: sequence type. 
and with 12 allele differences compared with the nearest strain of the regional cluster in Northern Finland (Table, Figure 1, 2 and 3). Case 19 was transferred from an Italian hospital and found positive by routine screening, case 20 by a clinical specimen. Both cases occupied the same room more than 8 months apart.

\section{Enhanced screening of patients and environment}

After identification of the first case (case 5) in HCFC, 17 specimens of nine potentially exposed patients were tested and all were negative. After identification of the second case in HCFC (case6), around 200 potentially exposed patients were screened in November 2015 and three new cases (case 8, 9 and 10) were found. The screening included discharged patients and two screening rounds in the haemodialysis unit and in one ward. Another ward was closed down for a week in December 2015 and 29 environmental specimens were subsequently taken, but all tested negative.

After detection of the third case in HCFD (case 15) 32 potentially exposed patients were screened and 16 environmental specimens were obtained in August 2017, all patient and environmental specimens tested negative.

HCFE screened 29 patients after detection of their second case (case 18) in March 2018 and obtained 46 environmental specimens, but all tested negative.

HCFA screened 26 patients and obtained 16 environmental specimens in March 2018 when the room occupied by several cases (case 1, 2, 3, 4, 13 and 14) was identified. All specimens tested negative.

After identification of the second case in HCFF (case 20) in April 2016, 54 potentially exposed patients treated in the same room as case 19 and 20 were flagged and specimens could be obtained from 28 of them; all tested negative. The hospital staff also performed two cross-sectional screening rounds in the same ward $(n=32)$, and obtained 142 environmental specimens. Seven environmental specimens tested positive for $K$. pneumoniae KPC-3 ST512, and were either identical or showed maximum three alleles differences compared with the strains found in specimens obtained from cases 19 and 20 (data not shown). The first specimens were obtained after ordinary cleaning (no disinfectant) of the patient room. The positive sites were the patient desk, windowsill, floor drain, toilet seat, and inner toilet surface nearby the water edge. Contact isolation and cleaning (peracetic acid/hydrogen peroxide) were performed in the room. In the control specimens the inner toilet surface remained positive and the sink trap in the room was a new positive site. Terminal cleaning (peracetic acid/hydrogen peroxide) was performed after the patient discharge, also thereafter the inner toilet surface remained positive and the floor drain was again positive. The toilet remained positive even after treatment with NOCOSPRAY (OXY'PHARM, Paris,
France) and with 1,000 ppm chlorine. Finally, it was negative after 2,000 ppm chlorine treatment and also in three control specimens (twice in May 2016 and once in March 2017).

\section{Discussion}

Here we describe two clusters of KPC-KP: one single hospital cluster with two cases and one regional cluster with 18 cases spread in five HCFs. Transfer of unknown carriers between HCFs and environmental contamination seemed to play a key role in KPC-KP transmission. The regional spread was initially not recognised due to transfer of previously unknown positive patients, long time intervals between successive cases, and distribution of cases in several HCFs.

The two clusters mainly affected acute care hospitals and most of the cases were detected by clinical specimens. Extensive screening of potentially exposed contact patients only identified a few new cases. Many of the patients were not only colonised by KPC-KP but had clinical infections, and one death was related to infection caused by KPC-KP. It also turned out that the disinfection control methods used previously were not effective in controlling the spread. The regional cluster is still ongoing in one HCF despite extensive measures including isolation of cases, screening, and enhanced environmental cleaning.

K. pneumoniae $\mathrm{ST}_{258}$, and related strains like $\mathrm{ST}_{512}$ with KPC carbapenemase gene as detected in this study, have caused healthcare-associated outbreaks globally since they were discovered in early 2000 and are considered to be endemic in HCFs in countries like China, Colombia, Greece, Israel, Italy, Puerto Rico, and the US $[19,20]$. In Finland, the first $K$. pneumoniae with KPC-2 gene was found in 2009 in a patient repatriated from Greece [21]. The first transfer of KPC-KP (ST258, $K P C-2)$ between two patients in Finland was confirmed in 2009 (data not shown) and the first outbreak caused by KPC-KP (ST512, KPC-3) occurred in 2013 [12]. In the previous outbreak, no direct link to travel abroad or environmental contamination was detected. The outbreak was successfully controlled by rapid and extensive screening together with cohorting and isolating carriers and exposed patients.

The core genome sequence analysis showed two distinct clusters with 12 allele differences, and we interpreted that both clusters were separate outbreaks. However, case 4 acquired 11 allele differences over the course of 2 years and 3 months, showing that analysis of WGS data using a strict cut-off needs to be complemented with epidemiological data for correct interpretation (as was done in this study). There was no patient transfer between HCFs of the two regions and the index case of the single hospital cluster was transferred from a hospital in Italy where KPC-KP is endemic. The origin of the regional cluster remained unknown. It is possible that undetected cases causing environmental contamination have previously existed. Notably, the index 
patient had negative surveillance cultures for MDR microbes before ward care during intensive care.

We found an epidemiological link (travel history, common ward or room) for most of the cases $(16 / 20)$. It is likely that we missed cases since epidemiological links for four cases were not found. It is unknown how the strain was transmitted to HCFC since we could not find an epidemiological link between the first case (case 5) of HCFC and other HCFs. We know that three patients were transferred from HCFA to HCFC before case 5 was detected. One of these three patients had shared a room with case 5 , this might have caused transmission between facilities (data not shown). This could not be verified since the patient died before the screening was performed.

Only two cases with overlapping stays in a common room were found in these clusters, supporting our hypothesis of environmental contamination and subsequent transmission to new cases. Outbreaks of $K$. pneumoniae with a persisting environmental reservoir and high resistance to cleaning efforts and as shown in HCFF in this study have been previously reported $[7,22$ 27]. Both cases of the single hospital cluster stayed in the same room more than 8 months apart, and the inanimate surfaces in the room and bathroom were found repeatedly positive, even after cleaning and decontamination. Although we think that most of the environmental contamination was caused by the second case since the specimens were taken after this case had stayed in the room, the long interval between the two cases and the efforts required for the decontamination suggest that the environment was the source of KPC-KP transmission. An alternative hypothesis could be that cases in this study did not obtain the strain from the environment, but directly from unknown carriers or indirectly via hands of healthcare workers.

Six cases could be linked to a common room in HCFA without overlapping stays within a period of 3 years and 1 month. This suggests that environmental transmission might have been the main transmission route in the regional cluster as well. Environmental specimens were obtained twice from this room, 2 months after the last positive case and tested negative, which could be a sign that the room has now been decontaminated. We, however, recommend to keep monitoring the room to circumvent the risk of false negative test results.

Both clusters were recognised by national surveillance using WGS, and stress the relevance of national CPE surveillance. Multiple interventions are needed to contain further spread. We could learn from Israel which has implemented and published a national strategy to contain a large outbreak of CPE [28]. Regional coordination needs to be implemented to actively monitor colonisation status of existing cases and trace-back transmission routes of new cases, especially when there is no link to hospitalisation abroad. Like our colleagues in Israel, we recommend that rooms occupied by CPE-positive patients be cleaned and disinfected once a day with dedicated, single-patient or single-use equipment [29]. Special attention should be paid to terminal cleaning following discharge, even to perform it twice by different teams and with different equipment. This applies to all CPE-positive patients, not only in outbreaks and independently of the test results of environmental specimens (positive/negative). If there is no link to a foreign country, the trace-back period for epidemiological investigations should be long enough to ensure finding all links. Sometimes more than 1 year may be needed. Since the yield in screening potentially exposed contact patients has constantly been small, we consider starting systematic screening of patients discharged from affected wards as a new strategy. The regional outbreak team needs to be supported by real-time genomic sequencing by the national reference laboratory and a regular feedback communication mechanism to the HCFs is required to stop further transmission.

\section{Acknowledgements}

This work was a team effort and we would like to acknowledge all people involved in the laboratory and epidemiological investigations. We would like to thank Ulla Kaukoniemi, infectious disease specialist, Länsi-Pohja Central Hospital, Finland, for support with the outbreak investigation. The authors wish to acknowledge CSC - IT Center for Science, Finland, for providing computational resources.

\section{Conflict of interest}

None declared.

\section{Authors' contributions}

Janko van Beek: outbreak investigation, preparation of table and figures, manuscript writing; Kati Räisänen: whole genome sequencing and data analysis, preparation of figures, manuscript writing; Markku Broas: outbreak investigation; Jari Kauranen: detection and characterisation of bacterial isolates; Arja Kähkölä: outbreak investigation; Janne Laine: outbreak investigation; Eeva Mustonen: outbreak investigation; Tuija Nurkkala: outbreak investigation; Teija Puhto: outbreak investigation; Jaana Sinkkonen: outbreak investigation; Senja Torvinen: outbreak investigation; Tarja Vornanen: outbreak investigation; Risto Vuento: detection of bacterial isolates, resistance typing; Jari Jalava: detection and characterisation of bacterial isolates, whole genome sequencing and data analysis, manuscript writing; Outi Lyytikäinen: outbreak investigation, manuscript writing, study design. All authors critically read and revised the drafts of the manuscript and approved the final version.

\section{References}

1. Tzouvelekis LS, Markogiannakis A, Psichogiou M, Tassios PT, Daikos GL. Carbapenemases in Klebsiella pneumoniae and other Enterobacteriaceae: an evolving crisis of global dimensions. Clin Microbiol Rev. 2012;25(4):682-707. https:// doi.org/10.1128/CMR.05035-11 PMID: 23034326

2. Munoz-Price LS, Poirel L, Bonomo RA, Schwaber MJ, Daikos GL, Cormican M, et al. Clinical epidemiology of the global expansion of Klebsiella pneumoniae carbapenemases. Lancet Infect Dis. 2013;13(9):785-96. https://doi.org/10.1016/S14733099(13)70190-7 PMID: 23969216 
3. European Centre for Disease Prevention and Control. Rapid risk assessment: Carbapenem-resistant Enterobacteriaceae - first update 4 June 2018. Stockholm: ECDC; 2018.

4. Hardiman CA, Weingarten RA, Conlan S, Khil P, Dekker JP, Mathers AJ, et al. Horizontal Transfer of CarbapenemaseEncoding Plasmids and Comparison with Hospital Epidemiology Data. Antimicrob Agents Chemother. 2016;6o(8):4910-9. https://doi.org/10.1128/AAC.00014-16 PMID: 27270289

5. Grundmann H, Glasner C, Albiger B, Aanensen DM, Tomlinson CT, Andrasević AT, et al. Occurrence of carbapenemaseproducing Klebsiella pneumoniae and Escherichia coli in the European survey of carbapenemase-producing Enterobacteriaceae (EuSCAPE): a prospective, multinational study. Lancet Infect Dis. 2017;17(2):153-63. https://doi. org/10.1016/S1473-3099(16)30257-2 PMID: 27866944

6. Errico G, Gagliotti C, Monaco M, Masiero L, Gaibani P, Ambretti $S$, et al. . Colonization and infection due to carbapenemaseproducing Enterobacteriaceae in liver and lung transplant recipients and donor-derived transmission: a prospective cohort study conducted in Italy. Clin Microbiol Infect. 2019;25(2):203-9. https://doi.org/10.1016/j.cmi.2018.05.003 PMID: 29800674

7. Snitkin ES, Zelazny AM, Thomas PJ, Stock F, Henderson DK, Palmore TN, et al. NISC Comparative Sequencing Program Group. Tracking a hospital outbreak of carbapenem-resistant Klebsiella pneumoniae with whole-genome sequencing. Sci Transl Med. 2012;4(148):148ra116. https://doi.org/10.1126/ scitranslmed.3004129 PMID: 22914622

8. Heinrichs A, Argudín MA, De Mendonça R, Deplano A, Roisin $S$, Dodémont M, et al. An Outpatient Çlinic as a Potentia Site of Transmission for an Outbreak of New Delhi Metallo- $\beta$ Lactamase-producing Klebsiella pneumoniae Sequence Type 716: A Study Using Whole-genome Sequencing. Clin Infect Dis. 2019;68(6):993-1000. https://doi.org/10.1093/cid/ciy581 PMID: 30032179

9. López-Camacho E, Paño-Pardo JR, Ruiz-Carrascoso G, Wesselink JJ, Lusa-Bernal S, Ramos-Ruiz R, et al. Population structure of OXA-48-producing Klebsiella pneumoniae ST405 isolates during a hospital outbreak characterised by genomic typing. J Glob Antimicrob Resist. 2018;15:48-54. https://doi. org/10.1016/j.jgar.2018.06.008 PMID: 29940334

10. Albiger B, Glasner C, Struelens MJ, Grundmann H, Monnet DLEuropean Survey of Carbapenemase-Producing Enterobacteriaceae (EuSCAPE) working group. Carbapenemaseproducing Enterobacteriaceae in Europe: assessment by national experts from 38 countries, May 2015. Euro Surveill. 2015;20(45):30062. https://doi.org/10.2807/1560-7917. ES.2015.20.45.30062 PMID: 26675038

11. Österblad M, Kirveskari J, Hakanen AJ, Tissari P, Vaara M, Jalava J. Carbapenemase-producing Enterobacteriaceae in Finland: the first years (2008-11). J Antimicrob Chemother. 2012;67(12):2860-4. https://doi.org/10.1093/jac/dks299 PMID: 22855858

12. Kanerva M, Skogberg K, Ryynänen K, Pahkamäki A, Jalava J, Ollgren J, et al. Coincidental detection of the first outbreak of carbapenemase-producing Klebsiella pneumoniae colonisation in a primary care hospital, Finland, 2013. Euro Surveill. 2015;20(26):21172. https://doi.org/10.2807/1560-7917. ES2015.20.26.21172 PMID: 26159309

13. Kolho E, Lyytikäinen O, Jalava J. Ohje moniresistenttien mikrobien tartunnantorjunnasta. [National guideline for control of multidrug-resistant microbes]. Helsinki: National Institute for Health and Welfare (THL); 2017. Finnish. Available from: http://urn.fi/URN:ISBN:978-952-302-943-9

14. Kluytmans-van den Bergh MF, Rossen JW, Bruijning-Verhagen PC, Bonten MJ, Friedrich AW, Vandenbroucke-Grauls CM, et al. Whole-Genome Multilocus Sequence Typing of ExtendedSpectrum-Beta-Lactamase-Producing Enterobacteriaceae. J Clin Microbiol. 2016;54(12):2919-27. https://doi.org/10.1128/ JCM.01648-16 PMID: 27629900

15. The European Committee on Antimicrobial Susceptibility Testing (EUCAST). Breakpoint tables for interpretation of MICs and zone diameters. Versions 3.1-8.1, 2013-2018. Växjö: EUCAST; 2018. Available from: http://www.eucast.org/ clinical_breakpoints/

16. Pasanen T, Koskela S, Mero S, Tarkka E, Tissari P, Vaara $M$, et al. Rapid molecular characterization of Acinetobacter baumannii clones with rep-PCR and evaluation of carbapenemase genes by new multiplex PCR in Hospital District of Helsinki and Uusimaa. PLoS One. 2014;9(1):e85854. https://doi.org/10.1371/journal.pone.0085854 PMID: 24465749

17. Jolley KA, Maiden MC. BIGSdb: Scalable analysis of bacterial genome variation at the population level. BMC Bioinformatics. 2010;11(1):595. https://doi.org/10.1186/1471-2105-11-595 PMID: 21143983
18. Inouye M, Dashnow H, Raven LA, Schultz MB, Pope BJ, Tomita T, et al. SRST2: Rapid genomic surveillance for public health and hospital microbiology labs. Genome Med. 2014;6(11):90. https://doi.org/10.1186/s13073-014-0090-6 PMID: 25422674

19. Pitout JD, Nordmann P, Poirel L. Carbapenemase-Producing Klebsiella pneumoniae, a Key Pathogen Set for Global Nosocomial Dominance. Antimicrob Agents Chemother. 2015;59(10):5873-84. https://doi.org/10.1128/AAC.01019-15 PMID: 26169401

20. Yigit H, Queenan AM, Anderson GJ, Domenech-Sanchez A, Biddle JW, Steward CD, et al. Novel carbapenem-hydrolyzing beta-lactamase, KPC-1, from a carbapenem-resistant strain of Klebsiella pneumoniae. Antimicrob Agents Chemother. 2001;45(4):1151-61. https://doi.org/10.1128/AAC.45.4.11511161.2001 PMID: 11257029

21. Osterblad M, Kirveskari J, Koskela S, Tissari P, Vuorenoja $\mathrm{K}$, Hakanen AJ, et al. First isolations of KPC-2-carrying ST258 Klebsiella pneumoniae strains in Finland, June and August 2009. Euro Surveill. 2009;14(40):19349. https://doi. org/10.2807/ese.14.40.19349-en PMID: 19822122

22. Tofteland S, Naseer U, Lislevand JH, Sundsfjord A, Samuelsen 0 . A long-term low-frequency hospital outbreak of KPCproducing Klebsiella pneumoniae involving Intergenus plasmid diffusion and a persisting environmental reservoir. PLoS One. 2013;8(3):e59015. https://doi.org/10.1371/journal. pone.0059015 PMID: 23536849

23. Pantel A, Richaud-Morel B, Cazaban M, Bouziges N, Sotto A, Lavigne JP. Environmental persistence of OXA-48-producing Klebsiella pneumoniae in a French intensive care unit. Am J Infect Control. 2016;44(3):366-8. https://doi.org/10.1016/j. ajic.2015.09.021 PMID: 26521704

24. Clarivet B, Grau D, Jumas-Bilak E, Jean-Pierre H, Pantel A, Parer $\mathrm{S}$, et al. Persisting transmission of carbapenemase-producing Klebsiella pneumoniae due to an environmental reservoir in a university hospital, France, 2012 to 2014. Euro Surveill. 2016;21(17):30213. https://doi.org/10.2807/1560-7917. ES.2016.21.17.30213 PMID: 27168586

25. Garvey MI, Bradley CW, Jumaa P. Environmental decontamination following occupancy of a burns patient with multiple carbapenemase-producing organisms. J Hosp Infect. 2016;93(2):136-40. https://doi.org/10.1016/j.jhin.2016.01.006 PMID: 26895617

26. Leitner E, Zarfel G, Luxner J, Herzog K, Pekard-Amenitsch S, Hoenigl $M$, et al. Contaminated handwashing sinks as the source of a clonal outbreak of KPC-2-producing Klebsiella oxytoca on a hematology ward. Antimicrob Agents Chemother. 2015;59(1):714-6. https://doi.org/10.1128/AAC.04306-14 PMID: 25348541

27. Carling PC. Wastewater drains: epidemiology and interventions in 23 carbapenem-resistant organism outbreaks. Infect Control Hosp Epidemiol. 2018;39(8):972-9. https://doi.org/10.1017/ ice.2018.138 PMID: 29950189

28. Solter E, Adler A, Rubinovitch B, Temkin E, Schwartz D, Ben-David D, et al. Israeli National Policy for CarbapenemResistant Enterobacteriaceae Screening, Carrier Isolation and Discontinuation of Isolation. Infect Control Hosp Epidemiol. 2018;39(1):85-9. https://doi.org/10.1017/ice.2017.211 PMID: 29241475

29. Friedman ND, Carmeli Y, Walton AL, Schwaber MJ. Carbapenem-Resistant Enterobacteriaceae: A Strategic Roadmap for Infection Control. Infect Control Hosp Epidemiol. 2017;38(5):580-94. https://doi.org/10.1017/ice.2017.42 PMID: 28294079

\section{License, supplementary material and copyright}

This is an open-access article distributed under the terms of the Creative Commons Attribution (CC BY 4.0) Licence. You may share and adapt the material, but must give appropriate credit to the source, provide a link to the licence and indicate if changes were made.

Any supplementary material referenced in the article can be found in the online version.

This article is copyright of the authors or their affiliated institutions, 2019. 